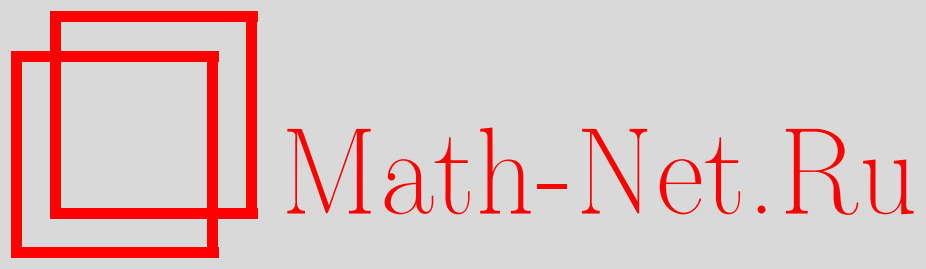

М. С. Агранович, Спектральные задачи в липшицевых областях для сильно эллиптических систем в банаховых пространствах $H_{p}^{\sigma}$ и $B_{p}^{\sigma}$, Функи. анализ и его прил., 2008, том 42, выпуск 4, 2-23

DOI: https://doi.org/10.4213/faa2933

Использование Общероссийского математического портала Math$\mathrm{Net.Ru}$ подразумевает, что вы прочитали и согласны с пользовательским соглашением

http://www. mathnet.ru/rus/agreement

Параметры загрузки:

IP : 54.210 .77 .194

26 апреля 2023 г., 17:55:24

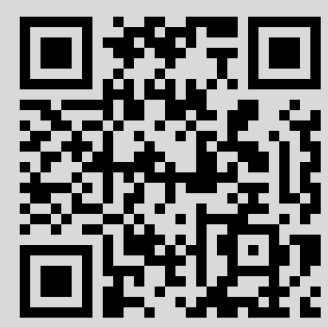


Функиионалъный анализ и его приложения

2008, т. 42, вып. 4, с. 2-23

УДК $517.98+517.95$

\title{
Спектральные задачи в липшицевых областях для сильно эллиптических систем в банаховых пространствах $H_{p}^{\sigma}$ и $B_{p}^{\sigma}$
}

\author{
(C) 2008. М. С. АГРАНович*
}

Дорогому Израилю Моисеевичу Гельфанду в связи с его 95-летием

\section{§1. Введение}

1.1. Постановка задач и основные утверждения. Для облегчения чтения мы будем рассматривать скалярное уравнение 2-го порядка до §6, где будут кратко объяснены обобщения. Оно имеет вид

$$
L u=\lambda u+f \quad \text { в } \Omega,
$$

где $L$ - оператор 2-го порядка для простоты вида

$$
L=-\sum \partial_{j} a_{j, k}(x) \partial_{k} u(x)+c(x) u(x)
$$

и $\Omega$ - ограниченная область в $\mathbb{R}^{n}, n \geqslant 2$, с липшицевой границей Г. Пример: уравнение $-\Delta u=\lambda u+f$. Коэффициенты $a_{j, k}(x)$ подчинены равномерному условию Липшица, коэффициент $c(x)$ - условию измеримости и ограниченности. Через $\partial_{k}$ обозначены частные производные $\partial / \partial x_{k}$. При желании можно включить в оператор $L$ и члены 1-го порядка. Матрица $A(x)=\left(a_{j, k}(x)\right)$ не предполагается вещественной или эрмитовой. Условие сильной эллиптичности [43] имеет вид

$$
\operatorname{Re} A(x)=\frac{1}{2}\left[A(x)+A^{*}(x)\right] \geqslant c_{0} I \quad(x \in \Omega),
$$

где $I$ - единичная матрица и $c_{0}$ - положительная постоянная. Это условие по непрерывности всегда можно усилить: при некотором $\theta>0$ и $|\psi| \leqslant \theta$

$$
\operatorname{Re}\left[e^{i \psi} A(x)\right] \geqslant c_{0}^{\prime} I \quad(x \in \Omega) .
$$

При исследовании граничных задач в липшицевых областях обычно используются потенциалы - объемный и поверхностные - и соответствующие интегральные уравнения. Наш же подход, как в [5] и [6], является чисто вариационным. Оператору (1.1.2) соответствует полуторалинейная форма

$$
\Phi(u, v)=\int_{\Omega} \sum a_{j k}(x) \partial_{k} u(x) \cdot \partial_{j} \overline{v(x)} d x+\int_{\Omega} c(x) u(x) \overline{v(x)} d x .
$$

Мы будем рассматривать задачи Дирихле и Неймана с однородными граничными условиями. Их вариационная (или слабая) постановка имеет вид

$$
\Phi(u, v)=\lambda(u, v)_{\Omega}+(f, v)_{\Omega},
$$

*Работа поддержана грантом РФФИ 07-01-00287. 
где $v$ - произвольная «пробная» функция. Сначала мы уточним эту постановку в пространствах потенциалов (или бесселевых потенциалов). В задаче Дирихле

$$
u \in \widetilde{H}_{p}^{1 / 2+s+1 / p}(\Omega), \quad f \in H_{p}^{-1 / 2+s-1 / p^{\prime}}(\Omega), \quad v \in \widetilde{H}_{p^{\prime}}^{1 / 2-s+1 / p^{\prime}}(\Omega) .
$$

Здесь и дальше

$$
|s|<1 / 2, \quad 1<p<\infty, \quad p p^{\prime}=p+p^{\prime} .
$$

Определения пространств $H_{p}^{\sigma}=H_{p}^{\sigma}\left(\mathbb{R}^{n}\right)$ и $H_{p}^{\sigma}(\Omega)$ мы напоминаем в п. 1.2. Через $\widetilde{H}_{p}^{\sigma}(\Omega)$ обозначено подпространство в $H_{p}^{\sigma}$, состоящее из элементов с носителями в замыкании $\bar{\Omega}$ области $\Omega$ (что означает подчинение решения однородным условиям Дирихле). В задаче Неймана

$$
u \in H_{p}^{1 / 2+s+1 / p}(\Omega), \quad f \in \widetilde{H}_{p}^{-1 / 2+s-1 / p^{\prime}}(\Omega), \quad v \in H_{p^{\prime}}^{1 / 2-s+1 / p^{\prime}}(\Omega) .
$$

Смысл последнего слагаемого в (1.1.6) также объясняется в п. 1.2.

Положим $t=1 / p$. Допустимые пары индексов $(s, t)$ - это точки квадрата

$$
Q=\{(s, t):|s|<1 / 2,0<t<1\} .
$$

Для этих задач удается доказать их однозначную разрешимость при $\operatorname{Re} \lambda=-\mu$, $\mu \geqslant \mu_{0}$ с достаточно большим $\mu_{0}$ в прямоугольнике

$$
Q_{\delta}=\{(s, t):|s|<1 / 2,|t-1 / 2|<\delta\},
$$

где число $\delta \in(0,1 / 2]$ зависит от оператора (1.1.2) и области $\Omega$ [6].

В центре квадрата $Q$ мы имеем вариационные задачи, лишь общностью отличающиеся от классических задач из учебников математической физики. Они однозначно разрешимы в силу леммы Вишика [43]-Лакса-Мильграма [25].

Определение (1.1.6) в более общих пространствах, чем в центре квадрата, встречается в литературе. Для нас оно является исходным. Но оно бесполезно вне центра, если нет дополнительных аналитических средств. У нас такими средствами являются метод Саваре исследования гладкости решений в липшицевых областях (см. [37] и [6]), использующий вариационную природу рассматриваемых задач и обобщающий метод разностных отношений Ниренберга [34], и средства теории интерполяции. Мы совсем не исполъзуем поверхностные потенииаль, что позволяет обойтись без нежелательных ограничений в случае задачи Неймана для систем. См. пояснения в [5], §4. С другой стороны, при помощи потенциалов и интегральных уравнений удается существенно расширить область $Q_{\delta}$ однозначной разрешимости в случае уравнения Бельтрами-Лапласа (см. [33]).

Сначала при $t=1 / 2,|s|<1 / 2$ доказываются теорема о гладкости решений (если решение отвечает точке $(s, t)$, а правая часть - точке $\left(s^{\prime}, t\right)$ с $s^{\prime}>s$, то решение отвечает точке $\left.\left(s^{\prime}, t\right)\right)$ и теорема об однозначной разрешимости. В случае самосопряженной вещественной матрицы $A(x)$ это теоремы Саваре [37], но они обобщаются не только на уравнения с несамосопряженными матрицами $A(x)$, но и на общие сильно эллиптические системы [6] при некоторых естественных дополнительных предположениях. Уже в методе Саваре используются тонкие средства теории интерполяции. Экстраполяция этих результатов на $(s, t) \in Q_{\delta}$ получена в [6] при помощи оценки Шнейберга из [39]. Однако все это результаты при фиксированном $\lambda$. 
Рассмотрим для определенности задачу Неймана с параметром $\lambda . \mathrm{B} \S 2$ мы выведем оценку вида

$$
\begin{aligned}
\|u\|_{H_{p}^{1 / 2+s+1 / p}(\Omega)} & =\|u\|_{H_{p}^{1 / 2+s+1 / p}(\Omega)}+\mu\|u\|_{\widetilde{H}_{p}^{-1 / 2+s-1 / p^{\prime}}(\Omega)} \\
& \leqslant C\|f\|_{\widetilde{H}_{p}^{-1 / 2+s-1 / p^{\prime}}(\Omega)},
\end{aligned}
$$

где $C$ не зависит от $u$ и $\lambda$. Здесь $\mu=-\operatorname{Re} \lambda>\mu_{0}$ с достаточно большим $\mu_{0}$. Это фактически оптимальная оценка резольвенты отвечающего задаче оператора $u \mapsto f$. Как следствие мы получим ее с $|\lambda|$ вместо $\mu$ для $\lambda$ из множества

$$
\Theta(r, \varphi)=\{\lambda:|\arg \lambda| \geqslant \varphi,|\lambda| \geqslant r\}
$$

с $\varphi>\pi / 2$. Но последнее ограничение снимается при условии (1.1.4).

Оценки аналогичного вида встречались в работах об общих «гладких» задачах, эллиптических с параметром, см., в частности, [1], [8], и аналогичных задачах в областях с границей, гладкой вне особых точек специального вида (см., например, [24]). Но соответствующие соображения в нынешней ситуации не работают, и результат выводится из анализа возможсностей, предоставляемых методом Саваре, теорией интерполяиии и оценкой Шнейберга.

Нужные нам общие теоремы о спектральных свойствах операторов хорошо известны для операторов в гильбертовых пространствах ([15], гл. XI, [27], [28]), но у нас при $p \neq 2$ пространства $H_{p}^{\sigma}$ и $B_{p}^{\sigma}$ негилъбертовы. В абстрактной форме спектральные задачи в банаховых пространствах рассмотрел А. С. Маркус [28], и из его теорем видно, что у нас должно получиться. Мы по существу и следуем его работе, но используем также более поздние публикации.

Маркус пользовался абстрактной теорией Гротендика [19], [20] операторных идеалов и определителей операторов в банаховых пространствах для представления резольвенты в виде отношения целых функций, привлекая дополнительно возможность перехода к степеням компактного оператора. Однако результаты этой теории долгое время оставались многовариантными и условными, хотя ее развитию было посвящено очень много работ. Намного позднее в литературе появились теоремы, которые позволяют добраться до нужных нам конкретных результатов. Хотя эти теоремы вошли в монографии ([23], [35] и др.), обилие определений и вариантов теории заставило автора решать нелегкую методическую задачу поиска, отбора, упрощения и соединения в единую цепочку нужных понятий и результатов. Эту абстрактную подоплеку наших спектральных задач в ее нынешнем состоянии мы излагаем в 33 и надеемся, что она интересна и сама по себе. ${ }^{1)}$ Ключевыми являются возможность систематического использования (только) аппроксимационных чисел и теорема 3.2.1 о представлении резольвенты в виде отношения целых аналитических функций с точной оценкой их роста. В конце параграфа приводятся и поясняются получающиеся на этой основе теоремы о полноте корневых функций и о суммируемости рядов Фурье по ним методом Абеля-Лидского ${ }^{2)}$, близкие к теоремам Данфорда-Шварца и Лидского в гильбертовом случае и к некоторым из теорем Маркуса в общем случае. В нашей редакции формулировки этих теорем максимально приближены к интересующим нас конкретным задачам и упрощены. Общий итог состоит

1) Автор очень признателен Р. С. Исмагилову, А. С. Маркусу и М. З. Соломяку за советы, которые помогли найти нужную литературу, и С. Майборода за полезные редакционные замечания.

2)В. Б. Лидский называл его методом Абеля. 
в том, что в рамках рассматриваемых здесь вопросов привычная в гильбертовом случае картина в основном распространяется на нужные нам банаховы пространства.

Следует отметить, что наши задачи несколько «лучше» абстрактных: это задачи в шкалах пространств с двумя индексами $(s, t)$, содержащих и сопряженные пространства, и при $t=1 / 2$ это гильбертовы шкалы. Спектр и корневые функции не зависят от $(s, t)$ (cp. [1]). Асимптотика собственных чисел известна в случае самосопряженных задач Дирихле и Неймана [11]. При $p=2$ полнота и суммируемость получаются из известных теорем для операторов в гильбертовых пространствах [15], [27], [28]. Утверждение о полноте можно распространить на остальные $(s, t) \in Q_{\delta}$ при помощи теорем вложения и изоморфизмов, определяемых оператором задачи. См. §4, где рассмотрена также задача со спектральным параметром в граничном условии; она немного сложнее. Однако наиболее полные результаты получаются на основе рассмотрений в 3 .

В пространствах Бесова $B_{p}^{\sigma}(\Omega)$ получаются такие же результаты, как в $H_{p}^{\sigma}(\Omega)$. Корневые функции принадлежат пересечению этих пространств.

Еще одно приложение оценки резольвенты - указание пространств, в которых однозначно разрешимы соответствующие нестационарные задачи, см. §5. Здесь мы пользуемся теоремами из теории параболических (или аналитических) полугрупп, см., например, [31], гл. 5, §4. Приложения к нестационарным задачам проще теоремы о суммируемости.

1.2. Функциональные пространства. Пространство $H_{p}^{\sigma}$ совпадает с соболевским пространством $W_{p}^{\sigma}$ при целых неотрицательных $\sigma$, а пространство $B_{p}^{\sigma}$ - с пространством Слободецкого $W_{p}^{\sigma}$ при нецелых положительных $\sigma$. На остальные $\sigma$ определения распространяются при помощи оператора

$$
\Lambda_{\tau}=F^{-1}\left(1+|\xi|^{2}\right)^{\tau / 2} F,
$$

где $\tau \in \mathbb{R}, F$ - преобразование Фурье. Этот оператор изоморфно действует в обеих шкалах, понижая верхний индекс на $\tau$, если $\tau>0$. Пространства $H^{\sigma}=$ $H_{2}^{\sigma}$ и $B_{2}^{\sigma}$ совпадают, и при $\sigma \geqslant 0$ они совпадают с $W_{2}^{\sigma}$.

Пространства $H_{p}^{\sigma}(\Omega)$ и $B_{p}^{\sigma}(\Omega)$ состоят из сужений функций (или обобщенных функций) из соответствующих пространств в $\mathbb{R}^{n}$ на $\Omega$ с обычной нормой inf. Важна сопряженность пространств $\widetilde{H}_{p}^{-\sigma}(\Omega)$ и $H_{p^{\prime}}^{\sigma}(\Omega)$, а также аналогичных пространств Бесова, относительно формы (мы выписываем ее для задачи Неймана)

$$
(f, v)_{\Omega}=(f, \mathscr{E} v)_{\mathbb{R}^{n}},
$$

где $\mathscr{E}$ - универсальный оператор продолжения функций из пространств в $\Omega$ до функций из соответствующих пространств в $\mathbb{R}^{n}[36]$. Функции $v$ и $f$ в $(1.1 .7)$ и (1.1.9) принадлежат сопряженным пространствам. В частности, эти пространства рефлексивны. Обобщенное неравенство Шварца имеет вид

$$
\left|(f, v)_{\Omega}\right| \leqslant C\|f\|_{\widetilde{H}_{p}^{-\sigma}(\Omega)}\|v\|_{H_{p^{\prime}}^{\sigma}(\Omega)}
$$

такое же неравенство верно в нормах пространств Бесова. См. [6], [7]. Эти пространства сепарабельны.

В области $\Omega$ оказываются нужными комбинированные шкаль (см. [42] и [7]). Для пространств потенциалов первая шкала состоит из пространств $H_{p}^{\sigma}(\Omega)$ при 
$\sigma<1 / p$ и пространств $\widetilde{H}_{p}^{\sigma}(\Omega)$ при $\sigma>-1 / p^{\prime} ;$ при $-1 / p^{\prime}<\sigma<1 / p$ эти пространства отождествляются, поэтому получается единая интерполяционная шкала для комплексного метода в силу теоремы Вольфа (см., например, [21]). Пространства этой шкалы сужаются с ростом $\sigma$. Она обслуживает задачу Дирихле. Вторая шкала, обслуживающая задачу Неймана, состоит из пространств $\widetilde{H}_{p}^{\sigma}(\Omega)$ при $\sigma<1 / p$ и $H_{p}^{\sigma}(\Omega)$ при $\sigma>-1 / p^{\prime}$. Это тоже интерполяционная шкала, в которой пространства сужаются с ростом $\sigma$. Аналогичные комбинированные шкалы составляются из пространств Бесова.

Отметим, что имеют место непрерывные вложения $H_{p}^{\sigma+\varepsilon} \subset B_{p}^{\sigma}$ и $B_{p}^{\sigma+\varepsilon} \subset H_{p}^{\sigma}$ или с $\varepsilon=0$, или, по крайней мере, со сколь угодно малым $\varepsilon>0$ (см., например, [41], п. 2.3.4).

В п. 4.2 нам понадобятся пространства $B_{p}^{\sigma}(\Gamma),|\sigma| \leqslant 1$. Нормы в них определяются через нормы в $\mathbb{R}^{n-1}$ при помощи разбиения единицы.

Приготовим оценку формы $\Phi(u, v)$. Пусть $u$ и $v$ - функции соответственно из $H_{p}^{1 / 2+1 / p+s}(\Omega)$ и $H_{p^{\prime}}^{1 / 2+1 / p^{\prime}-s}(\Omega)$. Тогда

$$
|\Phi(u, v)| \leqslant C\|u\|_{H_{p}^{1 / 2+1 / p+s}(\Omega)}\|v\|_{H_{p^{\prime}}^{1 / 2+1 / p^{\prime}-s}(\Omega)} .
$$

Действительно, $\left|(u, v)_{\Omega}\right| \leqslant C^{\prime}\|u\|_{H_{p}^{0}(\Omega)}\|v\|_{H_{p^{\prime}}^{0}(\Omega)}$, а при $c=0$

$$
|\Phi(u, v)| \leqslant \sum\left|\left(a_{j k} \partial_{j} u, \partial_{k} v\right)_{\Omega}\right| \leqslant C^{\prime \prime} \sum\left\|\partial_{j} u\right\|_{H_{p}^{1 / p-1 / 2+s}(\Omega)}\left\|\partial_{k} v\right\|_{H_{p^{\prime}}^{-1 / p+1 / 2-s}(\Omega)} ;
$$

здесь последний верхний индекс равен $1 / p^{\prime}-1 / 2-s$. Мы учли, что функции $a_{j k}$ липшицевы и что $-1 / p^{\prime}<\sigma<1 / p$ для $\sigma=1 / p-1 / 2+s$, поэтому $\widetilde{H}$ можно заменить на $H$. Отсюда сразу получается (1.2.4). Аналогичная оценка получается и в пространствах Бесова.

\section{§2. Оптимальные оценки резольвенты}

При наших обозначениях спектр оператора, отвечающего задаче Дирихле или Неймана, находится в правой полуплоскости плюс, возможно, окрестность начала. Наша цель сейчас будет состоять в выводе оценки (1.1.12) в левой полуплоскости. Для определенности мы продолжаем рассматривать задачу Неймана для скалярного уравнения 2-го порядка. Сначала в п. 2.1 мы уточним, что мы имеем в точке $(0,1 / 2)$ - центре квадрата $Q$. Эти результаты следует считать известными, по крайней мере, для классических задач. Затем в п. 2.2 мы рассмотрим все точки квадрата с $t=1 / 2$, используя метод Саваре. В этих двух пунктах мы сначала получим оценку старшей нормы решения с не зависящей от параметра постоянной, затем выведем из (1.1.6) оценку младшей нормы с параметром $\mu=-\operatorname{Re} \lambda$. Наконец, в п. 2.3 мы рассмотрим точки с $t$, близкими к $1 / 2$, используя оценку Шнейберга и некоторые детали процедур комплексной интерполяции. Через $C$ и $C_{i}$ будут обозначаться постоянные с нумерацией в пределах пункта; важно, что они не зависят не только от функций, но и от параметра $\lambda$.

2.1. Оценки в центре квадрата $\boldsymbol{Q}$. Положим

$\Psi_{\lambda}(u, v)=\operatorname{Re}\left[\Phi(u, v)-\lambda(u, v)_{\Omega}\right], \quad \Psi_{\mu}(u)=\Psi_{\lambda}(u, u)=\operatorname{Re} \Phi(u, u)+\mu(u, u)_{\Omega}$.

В отличие от [6] мы сейчас не включаем $\operatorname{Re}(f, u)_{\Omega}$ в последнюю форму. 
Из определения вариационного решения $u \in H^{1}(\Omega)$ (см. (1.1.6)) следует, что

$$
\Psi_{\lambda}(u, v)=\operatorname{Re}(f, v)_{\Omega} \quad\left(f \in \widetilde{H}^{-1}(\Omega), v \in H^{1}(\Omega)\right) .
$$

При $-\operatorname{Re} \lambda=\mu \geqslant \mu_{0}$ с достаточно большим $\mu_{0}$ мы располагаем неравенством

$$
\|u\|_{H^{1}(\Omega)}^{2} \leqslant C_{1} \Psi_{\mu}(u),
$$

поскольку условие (1.1.3) в случае скалярного уравнения обеспечивает коэрцитивность формы $\Psi_{\mu}$ на $H^{1}(\Omega)$. Существенно, что в (2.1.3) благодаря знаку $\mu$ постоянная $C_{1}$ не зависит от $\mu$. Согласно (1.2.4) мы имеем

$$
|\Phi(u, v)| \leqslant C_{2}\|u\|_{H^{1}(\Omega)}\|v\|_{H^{1}(\Omega)} .
$$

Из (2.1.2) при $u=v$ и (2.1.3) получаем $\|u\|_{H^{1}(\Omega)}^{2}+\mu\|u\|_{L_{2}(\Omega)}^{2} \leqslant C_{3} \operatorname{Re}(f, u)_{\Omega}$. Отсюда

$\|u\|_{H^{1}(\Omega)} \leqslant C_{4}\|f\|_{\widetilde{H}^{-1}(\Omega)}$ и, если $f \in L_{2}(\Omega), \quad \mu\|u\|_{L_{2}(\Omega)} \leqslant C_{5}\|f\|_{L_{2}(\Omega)}$.

В силу (2.1.1), (2.1.2) и (2.1.4) величина $\mu\|u\|_{\widetilde{H}^{-1}(\Omega)}$ не превосходит

$$
C_{6} \mu \sup _{v} \frac{\left|(u, v)_{\Omega}\right|}{\|v\|_{H^{1}(\Omega)}} \leqslant C_{6} \sup _{v} \frac{|\Phi(u, v)|+\left|(f, v)_{\Omega}\right|}{\|v\|_{H^{1}(\Omega)}} \leqslant C_{7}\left[\|u\|_{H^{1}(\Omega)}+\|f\|_{\widetilde{H}^{-1}(\Omega)}\right],
$$

что в силу первого из неравенств $(2.1 .5)$ дает $\mu\|u\|_{\widetilde{H}^{-1}(\Omega)} \leqslant C_{8}\|f\|_{\widetilde{H}^{-1}(\Omega)}$. Это нужная оценка резольвенты в точке $(0,1 / 2)$.

В итоге мы имеем в этой точке второе неравенство в (2.1.5) и неравенство

$$
\|u \mid\|_{H^{1}(\Omega)} \leqslant C_{9}\|f\|_{\widetilde{H}^{-1}(\Omega)} .
$$

Аналогичные оценки верны в подобластях области $\Omega$.

2.2. Оценки при $t=1 / 2$. В этом пункте мы пользуемся методом Саваре. Аргументация излагается конспективно; мы надеемся, что читатель будет иметь под рукой работы [37] и [6].

Ключевой момент состоит в следующем. Предполагая, что $f \in L_{2}(\Omega)$, нужно получить неравенство

$$
\|u\|_{B_{2, \infty}^{3 / 2}(\Omega)}^{2} \leqslant C_{1}\|f\|_{\widetilde{H}^{-1}(\Omega)}\|f\|_{L_{2}(\Omega)}
$$

(см. (3.26) в [6]). Слева - норма в соответствующем пространстве Бесова, см., например, [37] или [6].

Обозначим через $O_{\rho}$ открытый круг с центром в фиксированной граничной точке (малого) радиуса $\rho$ и через $\Omega_{\rho}$ его пересечение с областью $\Omega$. Пусть $\varphi(x)$ - бесконечно гладкая функция со значениями на $[0,1]$, равная 1 в $O_{\rho}$ и нулю вне $O_{2 \rho}$. Положим

$$
\mathscr{T}_{h} v(x)=\varphi(x) v(x+h)+[1-\varphi(x)] v(x) .
$$

Разность $\mathscr{T}_{h} v(x)-v(x)$ - это «локализованное приращение» функции $v(x)$. Сдвиг на $h$ в случае задачи Неймана происходит внутрь области $\Omega$.

Нам сейчас нужно получить для решения оценку (см. (3.13) в [6])

$$
\Psi_{\mu}\left(\mathscr{T}_{h} u\right)-\Psi_{\mu}(u) \leqslant C_{2}|h|\|u\|_{H^{1}\left(\Omega_{3 \rho}\right)}\left[\|u\|_{H^{1}\left(\Omega_{3 \rho}\right)}+\|f\|_{L_{2}\left(\Omega_{3 \rho}\right)}\right] .
$$


Она получается из следующих неравенств с использованием (2.1.5):

$$
\begin{gathered}
\Phi\left(\mathscr{T}_{h} u\right)-\Phi(u) \leqslant C_{3}|h|\|u\|_{H^{1}\left(\Omega_{3 \rho}\right)}^{2}, \\
\left(\mathscr{T}_{h} u, \mathscr{T}_{h} u\right)_{\Omega}-(u, u)_{\Omega} \leqslant C_{4}|h|\|\nabla u\|_{L_{2}\left(\Omega_{3 \rho}\right)}^{2}, \\
\left(f, \mathscr{T}_{h} u-u\right)_{\Omega} \leqslant C_{5}|h|\|f\|_{L_{2}\left(\Omega_{2 \rho}\right)}\|\nabla u\|_{L_{2}\left(\Omega_{3 \rho}\right)} .
\end{gathered}
$$

Это неравенства (3.21)-(3.23) из [6] (с $m=1)$; постоянные справа не зависят от $\lambda$, так как левые части не содержат $\lambda$. Неравенство (2.2.4) умножаем на $\mu$.

Действуя теперь, как в [6], мы можем при помощи интерполяции доказать обратимость оператора

$$
L_{\lambda}=L-\lambda I: H^{1+s}(\Omega) \rightarrow \widetilde{H}^{-1+s}(\Omega)
$$

$(0<s<1 / 2)$ с равномерной по $\lambda$ оценкой

$$
\|u\|_{H^{1+s}(\Omega)} \leqslant C_{6}\|f\|_{\widetilde{H}^{-1+s}(\Omega)} .
$$

Далее для получения оценки нормы с параметром действуем, как в предыдущем пункте. Согласно (1.2.4)

$$
|\Phi(u, v)| \leqslant C_{7}\|u\|_{H^{1+s}(\Omega)}\|v\|_{H^{1-s}(\Omega)} .
$$

Поэтому

$$
\begin{aligned}
\mu\|u\|_{\widetilde{H}^{-1+s}(\Omega)} & \leqslant C_{8} \mu \sup _{v} \frac{\left|(u, v)_{\Omega}\right|}{\|v\|_{H^{1-s}(\Omega)}} \leqslant C_{9} \sup _{v} \frac{|\Phi(u, v)|+\left|(f, v)_{\Omega}\right|}{\|v\|_{H^{1-s}}(\Omega)} \\
& \leqslant C_{10}\left[\|u\|_{H^{1+s}(\Omega)}+\|f\|_{\widetilde{H}^{-1+s}(\Omega)}\right] \leqslant C_{11}\|f\|_{\widetilde{H}^{-1+s}(\Omega)}
\end{aligned}
$$

с учетом (2.2.8) и (2.2.7). В итоге получаем нужную оценку

$$
\|u\|_{H^{1+s}(\Omega)} \leqslant C_{12}\|f\|_{\widetilde{H}^{-1+s}(\Omega)} .
$$

Для распространения результата на $s \in(-1 / 2,0)$ используем двойственность следующим образом. Пусть $s \in(0,1 / 2)$ и $u$ - решение уже рассмотренной задачи из $H^{1+s}(\Omega)$, так что для него мы уже имеем оценку $(2.2 .9)$ с не зависящей от $\lambda$ постоянной. Сейчас мы получим аналогичную оценку для принадлежащего $H^{1-s}(\Omega)$ решения $v$ задачи Неймана для уравнения $L^{*} v-\bar{\lambda} v=g$, где $L^{*}-$ оператор, формально сопряженный к $L$. Из определения вариационных решений следует, что $(f, v)_{\Omega}=(u, g)_{\Omega}$. Поэтому

$$
\begin{aligned}
\|v\|_{H^{1-s}(\Omega)} & \leqslant C_{13} \sup _{f} \frac{\left|(f, v)_{\Omega}\right|}{\|f\|_{\widetilde{H}^{-1+s}(\Omega)}} \leqslant C_{14} \sup _{f} \frac{\left|(u, g)_{\Omega}\right|}{\|f\|_{\widetilde{H}^{-1+s}(\Omega)}} \\
& \leqslant C_{15} \sup _{f} \frac{\|u\|_{H^{1+s}(\Omega)}\|g\|_{\widetilde{H}^{-1-s}(\Omega)}}{\|f\|_{\widetilde{H}^{-1+s}(\Omega)}} \leqslant C_{15}\|g\|_{\widetilde{H}^{-1-s}(\Omega)} .
\end{aligned}
$$

Ясно, что отсюда следует нужный результат.

2.3. Оценки при $\boldsymbol{t} \neq \mathbf{1 / 2}$. Теперь мы должны использовать результат Шнейберга [39]. Приведем его. Пусть $\left(E_{0}, E_{1}\right)$ и $\left(F_{0}, F_{1}\right)$ - пары банаховых пространств для простоты с непрерывными и плотными вложениями $E_{1} \subset E_{0}$, $F_{1} \subset F_{0}$. Пусть $\left\{E_{\alpha}\right\}$ и $\left\{F_{\alpha}\right\}(0 \leqslant \alpha \leqslant 1)-$ комплексные интерполяционные шкалы. (См., например, [9].) Далее, пусть $A$ - ограниченный оператор из $E_{0}$ в $F_{0}$ и его сужение на $E_{1}$ - ограниченный оператор из $E_{1}$ в $F_{1}$. Тогда сужение $A_{t}$ оператора $A$ на $E_{t}$ - ограниченный оператор из $E_{t}$ в $F_{t}, 0<t<1$. 
Пусть $M-$ максимальная из норм операторов $A_{0}$ и $A_{1}$. Если оператор $A_{t}$ имеет ограниченный обратный $B_{t}$, то через $\beta(t)$ обозначим норму последнего оператора. Предположим, что обратимость имеет место при $t=1 / 2$. Тогда она экстраполируется, а именно, она имеет место при

$$
q(t)<\left[M \beta\left(\frac{1}{2}\right)\right]^{-1}, \quad \text { где } q(t)=\operatorname{tg}\left[\frac{\pi}{2}\left|t-\frac{1}{2}\right|\right] .
$$

При этом

$$
\beta(t) \leqslant \frac{M \beta\left(\frac{1}{2}\right)-q(t)}{M\left[1-q(t) M \beta\left(\frac{1}{2}\right)\right]} .
$$

Операторы $B_{t}$ автоматически согласованы: если $t_{1}<t_{2}$, то $B_{t_{2}}=\left.B_{t_{1}}\right|_{F_{t_{2}}}$.

Результат Шнейберга используется следующим образом. Для любого $s$ на $(-1 / 2,1 / 2)$ рассматриваем вертикальный отрезок с серединой в точке $(s, 1 / 2)$ фиксированной длины, меньшей 1 . Нужно получить оптимальную оценку резольвенты при $|t-1 / 2|<\delta$ с некоторым $\delta>0$, не зависящим от $s$, равномерную по $\lambda$.

Но для этого надо показать, что мы можем воспользоваться приведенным результатом, рассматривая нормы, зависящие от параметра. С этой целью обратимся к доказательству теорем об интерполяции. См. [9], мы в основном ограничимся ссылками на эту книгу.

В теории пространств потенциалов и Бесова, как известно, важную роль играет «диадическое» разбиение единицы (см., например, [9], п. 6.2):

$$
\sum_{k=0}^{\infty} \varphi_{k}(\xi) \equiv 1 \quad \text { при } \xi \neq 0 .
$$

Здесь $\operatorname{supp} \varphi_{k}$ лежит в шаровом слое $\left\{\xi: 2^{k-1} \leqslant|\xi| \leqslant 2^{k+1}\right\}$ при $k \geqslant 1$. Положим $\psi_{k}(x)=F^{-1} \varphi_{k}(\xi)$. Любую функцию $u$ из $\mathscr{S}$ (пространство Шварца быстро убывающих бесконечно гладких функций) можно представить в виде

$$
u(x)=\sum_{k=0}^{\infty}\left(\psi_{k} * u\right)(x) .
$$

Это верно и для функций (или обобщенных функций) $u$ из $H_{p}^{\sigma}$.

Пространство $H_{p}^{\sigma}$ является ретрактом пространства $L_{p}\left(l_{2}^{\sigma}\right)$. См. [9], п. 6.4. Здесь $l_{2}^{\sigma}$ - пространство числовых последовательностей $a=\left\{a_{k}\right\}_{0}^{\infty}$ с нормой

$$
\|a\|_{l_{2}^{\sigma}}=\left(\sum_{0}^{\infty}\left(2^{k \sigma}\left|a_{k}\right|\right)^{2}\right)^{1 / 2} .
$$

Пространство $L_{p}\left(l_{2}^{\sigma}\right)$ состоит из функций на $\mathbb{R}^{n}$ со значениями в $l_{2}^{\sigma}$, принадлежащих $L_{p}$. Норма в $L_{p}\left(l_{2}^{\sigma}\right)$ определяется равенством

$$
\|a(x)\|_{L_{p}\left(l_{2}^{\sigma}\right)}=\|\| a(x)\left\|_{l_{2}^{\sigma}}\right\|_{L_{p}} .
$$

Утверждение о ретракте означает, что имеются непрерывные линейные отображения $\mathscr{J}: H_{p}^{\sigma} \rightarrow L_{p}\left(l_{2}^{\sigma}\right)$ и $\mathscr{P}: L_{p}\left(l_{2}^{\sigma}\right) \rightarrow H_{p}^{\sigma}$, произведение $\mathscr{P} \mathscr{J}$ которых единичный оператор. Здесь $\mathscr{J} u(x)=a(x)=\left\{a_{k}(x)=\left(\psi_{k} * u\right)(x)\right\}$. Важно, что эти операторы не зависят от $\sigma$ и $p$.

Таким образом, мы имеем эквивалентность

$$
\|u(x)\|_{H_{p}^{\sigma}} \asymp\|a(x)\|_{L_{p}\left(l_{2}^{\sigma}\right)},
$$


здесь и дальше в том смысле, что отношение норм заключено между положительными постоянными, не зависящими от индексов и рассматриваемых функций. Нужная теорема об интерполяции пространств $H_{p}^{\sigma}$

$$
\left[H_{p_{0}}^{\sigma}, H_{p_{1}}^{\sigma}\right]_{\theta}=H_{p}^{\sigma} \quad\left(0<\theta<1, \frac{1}{p}=\frac{1-\theta}{p_{0}}+\frac{\theta}{p_{1}}\right)
$$

выводится из теоремы об интерполяции пространств $L_{p}$ функций со значениями в банаховом пространстве $\left(l_{2}^{\sigma}\right)$.

Положим теперь

$\|u(x)\|_{H_{p}^{\sigma}}=\|u(x)\|_{H_{p}^{\sigma}}+\mu\|u(x)\|_{H_{p}^{\sigma-2},},\|a(x)\|_{L_{p}\left(l_{2}^{\sigma}\right)}=\|\| a(x)\left\|_{l_{2}^{\sigma}}+\mu\right\| a(x)\left\|_{l_{2}^{\sigma-2}}\right\|_{L_{p}}$. Несложно проверить, что

$$
\|u(x)\|_{H_{p}^{\sigma}} \asymp\|a(x)\| \|_{L_{p}\left(l_{2}^{\sigma}\right)},
$$

где постоянные, ограничивающие отношение норм, не зависят и от $\mu$.

Результат переносится на пространства $H_{p}^{\sigma}(\Omega), \widetilde{H}_{p}^{\sigma}(\Omega)$ и на пространства комбинированных шкал. Ср. [41], пп. 1.2.4 и 1.17.

Отсюда следует, что при интерполяции по вертикальным отрезкам получается нужный результат:

Теорема 2.3.1. При условии сильной эллиптичности существует такое $\delta \in(0,1 / 2]$ и такое $\mu_{0}>0$, что задача Неймана для уравнения (1.1.1) с правой частъю из $\widetilde{H}_{p}^{-1 / 2+s-1 / p^{\prime}}(\Omega)$ имеет для $\mu=-\operatorname{Re} \lambda>\mu_{0}$ одно и только одно решение при $|s|<1 / 2,|t-1 / 2|<\delta$ с априорной оченкой

$$
\|u\|_{H_{p}^{1 / 2+s+1 / p}(\Omega)} \leqslant C\|f\|_{\widetilde{H}_{p}^{-1 / 2+s-1 / p^{\prime}}(\Omega)},
$$

где постоянная не зависит от $и$ и $\lambda$.

Фактически оценка двусторонняя: норма справа оценивается через норму слева в силу (1.1.6), (1.2.3) и (1.2.4).

2.4. Случай задачи Дирихле. Все происходит точно так же, только норма с параметром решения определяется равенством

$$
\|u\|\left\|_{\widetilde{H}_{p}^{1 / 2+s+1 / p}(\Omega)}=\right\| u\left\|_{\widetilde{H}_{p}^{1 / 2+s+1 / p}(\Omega)}+\mu\right\| u \|_{H_{p}^{-1 / 2+s-1 / p^{\prime}}(\Omega)},
$$

а априорная оценка имеет вид

$$
\|u\|\left\|_{\widetilde{H}_{p}^{1 / 2+s+1 / p}(\Omega)} \leqslant C\right\| f \|_{H_{p}^{-1 / 2+s-1 / p^{\prime}}(\Omega)} .
$$

Снова получается двусторонняя оценка.

2.5. Оценки в пространствах Бесова. В этих пространствах получаются аналогичные результаты. Здесь существенно, что при $p=2$ эти пространства совпадают с пространствами потенциалов.

В пространстве $B_{p}^{\sigma}$ можно пользоваться следующей нормой (это $l_{p}^{\sigma}$-норма последовательности $\left\{\left\|a_{k}(x)\right\|_{L_{p}}\right\}$ и $l_{p}^{\sigma}\left(L_{p}\right)$-норма последовательности $\left.a(x)\right)$ :

$$
\|u\|_{B_{p}^{\sigma}} \asymp\|a(x)\|_{l_{p}^{\sigma}\left(L_{p}\right)}=\left|\sum_{k=0}^{\infty}\left[2^{k \sigma}\left\|\psi_{k} * u\right\|_{L_{p}}\right]^{p}\right|^{1 / p} .
$$

В [9], п. 5.6.3, содержится формула (с такими же $\theta$ и $p$, как в (2.3.8))

$$
\left[l_{p_{0}}^{\sigma}\left(L_{p_{0}}\right), l_{p_{1}}^{\sigma}\left(L_{p_{1}}\right)\right]_{\theta}=l_{p}^{\sigma}\left(L_{p}\right) .
$$


Пространство $B_{p}^{\sigma}$ является ретрактом пространства $l_{p}^{\sigma}\left(L_{p}\right)$. Отображения и $\mathscr{P}$ - те же самые. Этим путем получается теорема об интерполяции пространств Бесова, аналогичная (2.3.8).

Рассмотрим теперь нормы с параметром $\mu$ :

$$
\begin{aligned}
\|\| u(x) \|_{B_{p}^{\sigma}} & =\|u(x)\|_{B_{p}^{\sigma}}+\mu\|u(x)\|_{B_{p}^{\sigma-2}}, \\
\|a(x)\|_{l_{p}^{\sigma}\left(L_{p}\right)} & =\|a(x)\|_{l_{p}^{\sigma}\left(L_{p}\right)}+\mu\|a(x)\|_{l_{p}^{\sigma-2}\left(L_{p}\right)} .
\end{aligned}
$$

Легко видеть, что они эквивалентны:

$$
\|u(x)\|_{B_{p}^{\sigma}} \asymp \mid\|a(x)\|_{l_{p}^{\sigma}\left(L_{p}\right)}
$$

с оценками отношения норм, не зависящими и от $\mu$. Отсюда следует нужный нам результат об интерполяции по вертикальным отрезкам. В итоге получаем, что теорема типа теоремы 2.3.1 верна и для пространств Бесова.

Мы не рассматриваем общие пространства Бесова $B_{p q}^{\sigma}$ и Трибеля-Лизоркина $F_{p q}^{s}$, хотя это по-видимому возможно. (У нас $B_{p}^{s}=B_{p p}^{s}$ и $H_{p}^{s}=F_{p 2}^{s}$, см. [41].)

Отметим, что в работе [38] получены оценки резольвенты в $L_{p}$ для задачи Дирихле в случае сильно эллиптической системы с постоянными коэффициентами и симметричной вещественной главной частью при помощи потенциалов.

\section{§3. Спектральные задачи в банаховых пространствах}

3.1. Аппроксимационные числа. Пусть $X_{1}$ и $X_{2}$ - (бесконечномерные) банаховы пространства и $T-$ компактный оператор из $X_{1}$ в $X_{2}$. Для $T$ определены аппроксимационные числа $a_{k}(T), k=1,2, \ldots$ Для краткости будем называть их $а$-числами. Они определяются следующим образом:

$$
a_{k}(T)=\inf \|T-K\|, \quad \text { где } \operatorname{dim} K<k .
$$

Отметим некоторые свойства этих чисел (см., например, [23], [35], [16], [17]).

$1^{\circ} .\|T\|=a_{1}(T) \geqslant a_{2}(T) \geqslant \ldots$.

$2^{\circ}$. Для двух компактных операторов $S, T$ из $X_{1}$ в $X_{2}$

$$
a_{k+l-1}(S+T) \leqslant a_{k}(S)+a_{l}(T) .
$$

$3^{\circ}$. Если $S$ и $R$ - ограниченные операторы и произведение $S T R$ имеет смысл, то

$$
a_{k}(S T R) \leqslant\|S\| a_{k}(T)\|R\| .
$$

$4^{\circ}$. Если произведение $S T$ компактных операторов имеет смысл, то

$$
a_{k+l-1}(S T) \leqslant a_{k}(S) a_{l}(T) .
$$

$5^{\circ}$. Отсюда следует, что при $\gamma_{1}>0, \gamma_{2}>0$

$$
a_{k}(S)=O\left(k^{-\gamma_{1}}\right), a_{k}(T)=O\left(k^{-\gamma_{2}}\right) \Longrightarrow a_{k}(S T)=O\left(k^{-\gamma_{1}-\gamma_{2}}\right) .
$$

$6^{\circ}$. Если $T$ - оператор размерности $m$, то $a_{k}(T)=0$ при $k>m$.

$7^{\circ} . a_{k}(T)=a_{k}\left(T^{*}\right)$. См. [16], с. 55 .

Если пространства $X_{1}$ и $X_{2}$ гильбертовы, то $a$-числа совпадают с сингулярными числами $s_{k}(T)$ - собственными значениями оператора $\sqrt{T T^{*}}$ (или, что то же, $\left.\sqrt{T^{*} T}\right)$. В общих банаховых пространствах ничего похожего нет. 
Пусть $X_{1}=X_{2}$. Будем обозначать через $\lambda_{k}(T)(k=1,2, \ldots)$ все ненулевые собственные значения оператора $T$, занумерованные в порядке невозрастания их модулей с учетом кратностей.

$8^{\circ}$. При любом $q>0$ справедливо неравенство типа Вейля

$$
\sum_{1}^{\infty}\left|\lambda_{k}(T)\right|^{q} \leqslant C_{q} \sum_{1}^{\infty} a_{k}(T)^{q} .
$$

В гильбертовых пространствах оно было давно известно, а для банаховых пространств доказано Кёнигом в 1978 г. (см. [23], с. 85), намного позднее работы [28]; оно было усилением результата из [29].

Через $\mathscr{A}_{q}, q>0$, обозначим операторный идеал, определяемый условием

$$
\sum_{1}^{\infty} a_{k}(T)^{q}<\infty .
$$

В гильбертовом случае (иногда и в банаховом) в литературе используется обозначение $\mathfrak{S}_{q}$. Для операторов из $\mathscr{A}_{q}$ мы имеем сходимость ряда из $\left|\lambda_{k}(T)\right|^{q}$. Корень степени $q$ из левой части - квазинорма в $\mathscr{A}_{q}$.

$$
\left.9^{\circ} . a_{k}(T)=O\left(k^{-\gamma}\right) \Longrightarrow \lambda_{k}(T)=O\left(k^{-\gamma}\right) \text { (здесь } \gamma>0\right) \text {. См. [16], с. } 7 .
$$

Для компактных операторов в банаховых пространствах имеются и другие системы «s-чисел», а также система энтропийных чисел $e_{k}(T)$; но для нас именно $a$-числа наиболее удобны.

В наших приложениях мы будем иметь сепарабельные банаховы пространства $X$ и $Y$ с компактным и плотным вложением $Y$ в $X$. Задан оператор $\mathscr{T}$, действующий ограниченным образом из $X$ в $Y$, поэтому в $X$ действует компактный оператор $T=J \mathscr{T}$, где $J$ - оператор вложения $Y$ в $X$. Для простоты примем, что оператор $\mathscr{T}$ отображает $X$ на $Y$ изоморфно. Обратный оператор из $Y$ в $X$ обозначим через $\mathscr{L}$, а через $L-$ неограниченный оператор в $X$ с областью определения $Y$, действующий как $\mathscr{L}: L y=\mathscr{L} y$. Оператор $L$ замкнут и имеет дискретный спектр, состоящий из собственных значений $1 / \lambda_{k}(T)$.

Нам понадобится оператор $(I-\lambda T)^{-1}$. Это мероморфная операторнозначная функция от $\lambda$ с полюсами в точках $1 / \lambda_{k}(T)$. Резольвента Фредгольма компактного оператора $T$ и резольвента неограниченного оператора $L$

$$
R_{T}^{(f)}(\lambda)=T(I-\lambda T)^{-1}, \quad R_{L}(\lambda)=(L-\lambda I)^{-1}
$$

совпадают.

Именно для оператора $J$ мы сможем предположить известной оценку $a_{k}(J)=$ $O\left(k^{-\gamma}\right)$, как следствие она получается для оператора $T$ и оказывается равномерной по операторам $\mathscr{T}$ с единичной нормой.

Точные оценки $a$-чисел для вложений конкретных пространств типа Соболева восходят к работе Бирмана-Соломяка [10] о кусочно-полиномиальных приближениях функций, см. также [12]. Несколько иначе действовали Эдмундс и Трибель, получившие следующий результат для областей с бесконечно гладкой границей (1989-92, см., например, [17], п. 3.3.4). На липшицевы области он распространяется автоматически благодаря наличию универсального оператора продолжения. 
Предложение 3.1.1 (Эдмундс, Трибель). Пусть $\sigma_{1}<\sigma_{2} u \gamma=\left(\sigma_{2}-\sigma_{1}\right) / n$. Тогда для пары $X=H_{p}^{\sigma_{1}}(\Omega), Y=H_{p}^{\sigma_{2}}(\Omega)$ и аналогичной пары пространств Бесова $a_{k}(J)=O\left(k^{-\gamma}\right)$.

Пусть теперь $\sigma_{1}<0<\sigma_{2}, \gamma=\left(\sigma_{2}-\sigma_{1}\right) / n$, а $X$ и $Y$ - одна из следующих четырех пар: 1) $\left.\left.\widetilde{H}_{p}^{\sigma_{1}}(\Omega), H_{p}^{\sigma_{2}}(\Omega), 2\right) H_{p}^{\sigma_{1}}(\Omega), \widetilde{H}_{p}^{\sigma_{2}}(\Omega) ; 3\right)$ и 4) - аналогичные пары пространств Бесова.

Предложение 3.1.2. В случаях 1)-4) $a_{k}(J)=O\left(k^{-\gamma}\right)$.

Объясним доказательство на примере первой пары пространств. Представим оператор $J$ в виде $J=J_{2} J_{1}$, где $J_{1}$ - оператор вложения пространства $H_{p}^{\sigma_{2}}(\Omega)$ в $H_{p}^{0}(\Omega)=L_{p}(\Omega)=\widetilde{H}_{p}^{0}(\Omega)$ и $J_{2}$ - оператор вложения пространства $\widetilde{H}_{p}^{0}(\Omega)$ в $\widetilde{H}_{p}^{\sigma_{1}}(\Omega)$. Мы имеем $a_{k}\left(J_{1}\right)=O\left(k^{-\sigma_{2} / n}\right)$ в силу предложения 3.1.1. Оператор $J_{2}$ является сопряженным к оператору вложения $H_{p}^{\left|\sigma_{1}\right|}(\Omega)$ в $H_{p}^{0}(\Omega)$ (относительно формы вида (1.2.2)). Поэтому $a_{k}\left(J_{2}\right)=O\left(k^{\sigma_{1} / n}\right)$ в силу того же предложения и $7^{\circ}$. Отсюда получаем $a_{k}(J)=O\left(k^{-\gamma}\right)$ в силу $5^{\circ}$.

3.2. Определители и представление резольвенты. Сначала предположим, что оператор $T$ в $X$ принадлежит $\mathscr{A}_{q}$ с $q \leqslant 1$. Тогда мы можем составить спектральный определитель (с $\left.\lambda_{j}=\lambda_{j}(T)\right)$

$$
d_{T}(\lambda)=d(I-\lambda T)=\prod_{1}^{\infty}\left(1-\lambda \lambda_{j}\right) .
$$

Известно и элементарно проверяется, что это целая аналитическая функция от $\lambda$ с ростом не выше чем порядка $q$ и минимального типа (ср. с теоремой 7 в [26], гл. I):

$$
\left|d_{T}(\lambda)\right| \leqslant C_{\varepsilon} \exp \left(\varepsilon|\lambda|^{q}\right)
$$

при любом $\varepsilon>0$. Проблемой, однако, оказывается нужное нам дальше мулътипликативное свойство (очевидное для конечномерных $S$ и $T$ )

$$
d(I-T) d(I-S)=d(I-(T+S-T S))
$$

(см. [35], с. 194). С другой стороны, в литературе имеется восходящее к Гротендику и использованное в [28] аксиоматическое определение определителя $d(I-T)$. См. [35], с. 185. Аксиомы включают свойство (3.2.3). На некоторых операторных идеалах такой определитель оказывается единственным, если добавить некоторое предположение о непрерывности, а иногда это именно спектральный определитель (3.2.1). Счастливым образом именно так обстоит дело с идеалом $\mathscr{A}_{1}$. См. [35], с. 210. ${ }^{1)}$

В произведении

$$
\Delta_{T}(\lambda)=d_{T}(\lambda)(I-\lambda T)^{-1}
$$

первый множитель гасит все особенности второго, так что это операторнозначная целая аналитическая функция от $\lambda$. Мы имеем при $\lambda \neq 1 / \lambda_{j}(T)$

$$
(I-\lambda T)^{-1}=\frac{\Delta_{T}(\lambda)}{d_{T}(\lambda)}
$$

1) Может быть, по аналогии с гильбертовым случаем именно операторы из $\mathscr{A}_{1}$ следовало бы называть ядерными. Но у Гротендика и в дальнейших работах ядерные операторы определены иначе. Мы не пользуемся этим определением. 
и покажем, что норма числителя оценивается так же, как знаменатель. Тем самым мы докажем следующую теорему, пока при $0<q \leqslant 1$ :

Теорема 3.2.1. Пусть $T \in \mathscr{A}_{q}$. Тогда справедлива формула $(3.2 .5)$, где в знаменателе - целая аналитическая функиия с ростом не выше чем порядка $q$ и минимального типа, а в числителе - операторнозначная иелая аналитическая функиия с аналогичной ощенкой нормы: при любом $\varepsilon>0$

$$
\left\|\Delta_{T}(\lambda)\right\| \leqslant C_{\varepsilon}^{\prime} \exp \left(\varepsilon|\lambda|^{q}\right) .
$$

Доказательство для $\boldsymbol{q} \leqslant \mathbf{1}$. Оценим сначала билинейную форму оператоpa $\Delta_{T}(\lambda)$. Для этого выведем формулу типа формулы Мацаева в [28], с. 534. (В отношении гильбертова случая там приведены ссылки на [30] и [18], гл. 5, $\S 5$, п. 1. Ниже мы немного отклоняемся от [28].)

Обозначим через $\langle x, z\rangle$ значение функционала $z$ из сопряженного к $X$ пространства $X^{*}$ на элементе $x \in X$. Построим одномерное возмущение оператора $I-\lambda T$ следующим образом. Возьмем $x \in X, z \in X^{*}$. Одномерный оператор $\langle\cdot, z\rangle x$, конечно, тоже принадлежит $\mathscr{A}_{q}$. Рассмотрим оператор

$$
I-\lambda T+\lambda\langle\cdot, z\rangle x=(I-\lambda T)\left(I+\lambda\langle\cdot, z\rangle(I-\lambda T)^{-1} x\right) .
$$

В силу (3.2.3)

$$
\begin{aligned}
d(I-\lambda T+\lambda\langle\cdot, z\rangle x) & =d(I-\lambda T) d\left(I+\lambda\langle\cdot, z\rangle(I-\lambda T)^{-1} x\right) \\
& =d(I-\lambda T)\left(1+\lambda\left\langle(I-\lambda T)^{-1} x, z\right\rangle\right) .
\end{aligned}
$$

Здесь мы вычислили определитель одномерного оператора. Отсюда и из (3.2.5)

$$
\left\langle\Delta_{T}(\lambda) x, z\right\rangle=\lambda^{-1}[d(I-\lambda T+\lambda\langle\cdot, z\rangle x)-d(I-\lambda T)] .
$$

Это и есть формула типа Мацаева. Из нее с учетом $1^{\circ}, 2^{\circ}$ и $6^{\circ}$ видно, что

$$
\left|\left\langle\Delta_{T}(\lambda) x, z\right\rangle\right| \leqslant C_{\varepsilon}(x, z) \exp \left(\varepsilon|\lambda|^{q}\right) .
$$

Используя теорему Банаха-Штейнхауза, мы делаем вывод, что при любом $\varepsilon>0$ для любой последовательности чисел $\left\{\lambda^{(k)}\right\}$ оценка (3.2.6) верна при $\lambda=\lambda^{(k)}$ с постоянной $C_{\varepsilon}$, не зависящей от $k$. Теперь от противного проверяется, что неравенство вида (3.2.6) верно для всех $\lambda$.

Далее мы действуем в основном по известным стандартам, см. [15], гл. ХІ, п. 9, [27] и [28]. Отметим, что в теореме о суммируемости Лидский накладывал условия на область значений квадратичной формы оператора. Вместо этого Маркус предполагал наличие оценки резольвенты в секторе с вершиной в начале, а у Данфорда-Шварца в теореме о полноте в гильбертовом случае предполагалось наличие лучей с достаточно хорошей оценкой нормы резольвенты. Мы следуем [15] и [28], но результаты получим в обоих пространствах, $X$ и $Y$. В отношении доказательства теоремы о суммируемости в [28] указано лишь, что оно аналогично доказательству Лидского. Мы приведем дополнительные подробности; дальнейшие детали в гильбертовом случае можно смотреть также в [2], §35.1) Мы не будем приводить возможные варианты теорем о полноте и суммируемости, см. по этому поводу [28].

3.3. Случай $\boldsymbol{q}>$ 1. В этом случае нужна регуляризация дроби в (3.2.5). Проведем ее так (фактически следуя [28]; другой и более известный способ см.,

1) В этой работе пробелом является отсутствие ссылки на [28]. 
например, в [15] и [27]). Найдем такое натуральное $N$, что $N<q \leqslant N+1$. Так как $T^{N+1} \in \mathscr{A}_{q /(N+1)}$ (это легко выводится из $\left.4^{\circ}\right)$, то можно пользоваться спектральным определителем $d_{T^{N+1}}(\lambda)$. Далее, $d_{T^{N+1}}\left(\lambda^{N+1}\right)$ - целая функция от $\lambda$ с ростом не выше чем порядка $q$ и минимального типа. Из тождества

$$
\left(I+\lambda T+\ldots+\lambda^{N} T^{N}\right)(I-\lambda T)=I-\lambda^{N+1} T^{N+1}
$$

следует, что при $\lambda^{N+1} \neq 1 / \lambda_{j}\left(T^{N+1}\right)$ мы имеем

$$
(I-\lambda T)^{-1}=\frac{\Delta_{T, N}(\lambda)}{d_{T^{N+1}}\left(\lambda^{N+1}\right)},
$$

где числитель

$$
\Delta_{T, N}(\lambda)=d_{T^{N+1}}\left(\lambda^{N+1}\right)\left(I-\lambda^{N+1} T^{N+1}\right)^{-1}\left(I+\ldots+\lambda^{N} T^{N}\right)
$$

имеет «лишние» нули там же, где знаменатель. Для формы $\left\langle\Delta_{T, N}(\lambda) x, z\right\rangle$ получается выражение типа (3.2.9)

$$
\lambda^{-N-1}\left[d\left(I-\lambda^{N+1} T^{N+1}+\lambda^{N+1}\langle\cdot, z\rangle\left(I+\ldots+\lambda^{N} T^{N}\right) x\right)-d\left(I-\lambda^{N+1} T^{N+1}\right)\right] .
$$

Отсюда для $\Delta_{T, N}(\lambda)$ получается оценка вида (3.2.6). Таким образом, теорема 3.2.1 распространяется на $q>1$ со следующим соглашением: в этом случае под дробью (3.2.5) будем понимать дробь (3.3.1).

Замечания. 1. Если умножить на $T$ левую часть формулы (3.2.5) (или (3.3.1)) и числитель в правой части, то получается представление резольвенты $R_{L}(\lambda)$ с сохранением оценки вида (3.2.6) для числителя.

2. Используя $9^{\circ}$, заменим предположение $T \in \mathscr{A}_{q}$ условием $a_{k}(T)=O\left(k^{-\gamma}\right)$, $\gamma>0$. Тогда справедлива формула типа (3.2.5) (или (3.3.1)), в которой знаменатель - иелая функиия с ростом не выше чем порядка $1 / \gamma$ и нормального типа, а числитель - операторнозначная целая функиия с аналогичной оченкой нормы.

Поясним, что $a_{k}=O\left(k^{-\gamma}\right)$ влечет $T \in \mathscr{A}_{q}$ при $q>1 / \gamma$. Сказанное про знаменатель $d_{T}(\lambda)$ при $\gamma>1-$ снова известный факт из теории целых функций (см. теорему 7 в [26], гл. I), а при $N \leqslant 1 / \gamma<N+1$ то же верно для $d_{T^{N+1}}\left(\lambda^{N+1}\right)$. Оценка для числителя в обоих случаях получается тем же путем, что и выше.

3. После того как настоящая работа была закончена, автор нашел в литературе работу [14]. В ней доказывается теорема типа 3.2.1 для оператора с $\sum c_{k}^{p}(T)<\infty$, где $c_{k}(T)$ - так называемые числа Гельфанда. Доказательство использует комбинацию результатов из [35] (есть также ссылки на [23] и [16]), но оно тяжелее нашего. Аппроксимационные числа мажорируют числа Гельфанда: $a_{k}(T) \geqslant c_{k}(T)$. Результат при $\sum c_{k}(T)^{q}<\infty$ немного слабее нашего при $\sum a_{k}(T)^{q}<\infty$ (нормальный тип вместо минимального). Наша теорема о полноте в следующем пункте близка к теореме из [14] (и обе близки к теоремам из [28]). Она все же оставлена в тексте для полноты изложения.

\section{4. Теорема о полноте.}

Теорема 3.4.1. Пусть $T \in \mathscr{A}_{q}$. Предположим, что имеются выходящие из начала лучи $\Gamma_{1}, \ldots, \Gamma_{K}$, вдоль которых резольвента $R_{L}(\lambda)$ существует при достаточно больших $|\lambda|$ и удовлетворяет соотношению

$$
\left\|R_{L}(\lambda)\right\|=O\left(|\lambda|^{h}\right)
$$


при некотором $h \in[-1,0)$. Пусть все угль между соседними лучами не больше $\pi / q$. Тогда система корневых векторов оператора $L$ полна в $X u Y$.

Доказательство. Предположим, что полноты нет в множестве векторов из $X$ вида $y=T x$. Тогда найдется такой вектор $z_{0} \in X^{*}$, что функционал $\left\langle\cdot, z_{0}\right\rangle$ аннулирует замкнутую линейную оболочку корневых векторов, но $\left\langle T x_{0}, z_{0}\right\rangle \neq 0$ при некотором $x_{0} \in X$. Рассмотрим функцию

$$
F(\lambda)=\left\langle R_{L}(\lambda) x_{0}, z_{0}\right\rangle .
$$

У нее нет полюсов, так что это целая аналитическая функция. По известной теореме из теории аналитических функций дробь

$$
\left(R_{L}(\lambda) x_{0}, z_{0}\right)=\frac{\left(\Delta_{T}(\lambda) T x_{0}, z_{0}\right)}{d_{T}(\lambda)}
$$

оказывается тогда целой аналитической функцией с ростом не выше чем порядка $q$ и минимального типа (см. [26], гл. I, §9). По теореме Фрагмена-Линделёфа (см., например, там же, §14) в сочетании с теоремой Лиувилля это постоянная и, значит, тождественный нуль. Полагая $\lambda=0$, получаем $\left\langle T x_{0}, z_{0}\right\rangle=0$, что противоречит предположению.

Так как векторы $T x$ плотны в $X$, то полнота имеет место в $X$. В силу изоморфизма она имеет место и в $Y$ в норме этого пространства.

От условия $h<0$ легко освободиться, используя степени оператора $T$.

3.5. Теорема о суммируемости. Определение метода Абеля-Лидского мы напомним немного ниже. Примем, что $T \in \mathscr{A}_{q}$ и оченка

$$
\left\|R_{L}(\lambda)\right\| \leqslant C(1+|\lambda|)^{-1}
$$

имеет место в (замкнутом) секторе $\Theta(r, \varphi)$ (см. (1.1.13)), где

$$
2 q \varphi<\pi
$$

Пусть для простоты $r=0$ : этого всегда можно добиться сдвигом спектрального параметра. Составим интеграл

$$
x(\tau)=(2 \pi i)^{-1} \int_{\Gamma_{\Theta}} \exp \left(-\tau \lambda^{q}\right) R_{L}(\lambda) d \lambda x \quad(\tau>0)
$$

по контуру $\Gamma_{\Theta}$ сектора $\Theta(0, \varphi)$ из бесконечности снизу вверх в бесконечность. Здесь и дальше $\lambda^{q}=|\lambda|^{q} \exp (i q \arg \lambda),|\arg \lambda| \leqslant \varphi$. Этот интеграл абсолютно сходится в силу (3.5.1) и (3.5.2), так как $\cos (q \varphi)>0$.

Проверяется, что в норме пространства $X$

$$
\lim _{\tau \downarrow 0} x(\tau) x=x
$$

для любого $x$ сначала из $Y$. Для распространения результата на все $x \in X$ доказывается, что оператор $x \mapsto x(\tau)$ равномерно ограничен при малых $\tau$.

Теперь интеграл $x(\tau)$ преобразуется в ряд из интегралов:

$$
x(\tau)=\sum_{k=1}^{\infty} x_{k}(\tau), \text { где } x_{k}(\tau)=(2 \pi i)^{-1} \int_{\Gamma_{k}} \exp \left(-\tau \lambda^{q}\right) R_{L}(\lambda) d \lambda x .
$$

Здесь $\Gamma_{k}$ - замкнутые контуры частей дополнения $\widetilde{\Theta}(0, \varphi)$ к сектору $\Theta(0, \varphi)$, на которые оно рассекается дугами $\Lambda_{k}$ окружностей с центром в начале радиусов $R_{k} \uparrow \infty$. Формулы (3.5.5) и (3.5.4), где в последней каждый интеграл $x_{k}(\tau)$ 
вычисляется при помощи вычетов, и определяют метод Абеля-Лидского поряд$\kappa a q$. Поясним, что интеграл $x_{k}(\tau)$ - линейная комбинация с экспоненциально зависящими от $\tau$ коэффициентами корневых векторов оператора $L$, отвечающих собственным значениям, окружаемым контуром $\Gamma_{k}$. Но проблема выбора радиусов $R_{k}$ нетривиальна.

Здесь используется теорема об оценке голоморфной функции снизу (см. [26], гл. I, §11, и [28], лемма 1.6), она применяется к знаменателю в (3.2.5) или (3.3.1). Если задать последовательность чисел $\varepsilon_{k} \downarrow 0$, то найдется такая последовательность $R_{k} \uparrow \infty$, что на дугах $\Lambda_{k} \subset \widetilde{\Theta}(0, \varphi)$ радиусов $R_{k}$ у оператора $L$ нет собственных значений и

$$
\left\|R_{L}(\lambda)\right\|_{X \rightarrow X} \leqslant \exp \left(\varepsilon_{k} R_{k}^{q}\right) .
$$

Проверяется, что тогда при $\tau>0$ по норме в $X$

$$
\lim _{k \rightarrow \infty} \int_{\Lambda_{k}} \exp \left(-\tau \lambda^{q}\right) R_{L}(\lambda) d \lambda x=0,
$$

что и приводит к нужному результату - сходимости ряда в (3.5.5) для $x \in X$.

Для векторов $x=L y$ мы имеем $\|y\|_{Y} \asymp\|x\|_{X}$. Заменим в (3.5.3)-(3.5.5) и (3.5.7) $x$ на $L y$. Оператор $L$ перестановочен с операциями в этих формулах. Поэтому для векторов $y \in Y$ результат верен в норме этого пространства.

Итак, получается

Теорема 3.5.1. При предположениях, указанных в начале пункта, ряд Фуръе любого вектора $x \in X$ по корневым векторам оператора $L$ суммируется $\kappa x$ методом Абеля-Лидского порядка q. Для $y \in Y$ это верно в норме пространства $Y$.

\section{§4. Спектральные задачи в липшицевых областях}

4.1. Спектральные задачи Дирихле и Неймана. Вернемся к задачам, рассмотренным в 2 . Независимость корневых функций отвечающего задаче оператора $L$ от $(s, t) \in Q_{\delta}$ (тем самым и собственных значений) устанавливается, как в [5], с использованием плотных вложений и изоморфизмов, определяемых этими задачами. Асимптотика спектра самосопряженных задач (в смысле $(L u, v)_{\Omega}=(u, L v)_{\Omega}$ на функциях из $\left.H^{1}(\Omega)\right)$ установлена, например, в [11]. Для обратного оператора $T$ в случае уравнений и систем 2-го порядка она имеет вид

$$
\lambda_{k}(T) \sim c k^{-2 / n},
$$

где $c=c(L, \Omega)$ - положительный коэффициент, одинаковый в задачах Дирихле и Неймана. В [11], правда, рассмотрена другая реализация оператора - в пространстве $L_{2}(\Omega)$ с областью определения, содержащейся в $H^{1}(\Omega)$. Но ее собственные функции остаются собственными функциями в нашем смысле; обе системы собственных функций полны в $L_{2}(\Omega)$ и минимальны, поэтому совпадают и собственные функции, и собственные значения. Из (4.1.1) уже для задач без предположения о самосопряженности следует аналогичная оценка $a$-чисел при $p=2: a_{k}=O\left(k^{-\gamma}\right)$ с $\gamma=2 / n$. Действительно, оператор общей задачи можно представить в виде произведения ограниченного оператора на оператор самосопряженной задачи. Это сразу приводит к теореме о полноте корневых функций в точке $(s, t)=(0,1 / 2)$ в предположении, что мы имеем оптимальную оценку 
резольвенты в $\Theta(r, \varphi)$ с $\varphi<\pi / n$. На остальные $(s, t) \in Q_{\delta}$ результат о полноте можно распространить, снова используя плотные вложения и определяемые операторами задач изоморфизмы, как в [5]. Сp. [1]. Он следует и из теоремы 3.4 .1 (в случае задачи Неймана с $X=\widetilde{H}_{p}^{-1 / 2+s-1 / p^{\prime}}(\Omega), Y=H_{p}^{1 / 2+s+1 / p}(\Omega)$ или аналогичными пространствами Бесова; в случае задачи Дирихле тильда переходит с первого на второе пространство). Далее, теорема о суммируемости при $p=2$ получается с $q \in(n / 2, \pi /(2 \varphi))$ из известной теоремы для операторов в гильбертовых пространствах. В общем случае мы используем теорему 3.5.1, а для ее получения - теорему 3.2.1.

Резюмируем окончательный результат.

Теорема 4.1.1. Корневые функиии и собственные значения задачи Неймана не зависят от $(s, t) \in Q_{\delta}$. Корневые функиии принадлежат пересечению пространств $H_{p}^{1 / 2+s+1 / p}(\Omega)$ и $B_{p}^{1 / 2+s+1 / p}(\Omega)$ для точек $(s, t) \in Q_{\delta}$. Собственные значения оператора $T$, обратного $\kappa$ оператору $L$ задачи, имеют оценку $\lambda_{k}=O\left(k^{-2 / n}\right)$. Oператор $(I-\lambda T)^{-1}$ представим в виде отношения иельх аналитических функиий, операторнозначной и числовой; функиия в знаменателе - роста не выше чем порядка n/2 и нормального типа; норма числителя имеет аналогичную оценку.

Пусть выполнено условие (1.1.4), обеспечивающее оптимальную оценку резольвенты в $\Theta(r, \varphi)$ с $\varphi<\pi / n$. Тогда во всех указанных пространствах $u$ пространствах $\widetilde{H}_{p}^{-1 / 2+s-1 / p^{\prime}}(\Omega)$ и $\widetilde{B}_{p}^{-1 / 2+s-1 / p^{\prime}}(\Omega)$ имеет место полнота корневых функиий, а при $n / 2<q<\pi /(2 \varphi)$ - суммируемость рядов Фуръе по ним методом Абеля-Лидского порядка $q$.

Для задачи Дирихле имеют место аналогичные результаты с заменой пространств $H$ и $B$ на $\widetilde{H}$ и $\widetilde{B}$ инноборот. ${ }^{1)}$

\section{2. Задачи со спектральным параметром в граничном условии.} Предположим, что задачи Дирихле и Неймана для уравнения $L u=f$ однозначно разрешимы для $(s, t) \in Q_{\delta}$. Относительно их вариационной постановки при неоднородных граничных условиях см., например, [5], [6]. Рассмотрим задачу

$$
L u=0 \quad \text { в } \Omega, \quad \partial_{\nu_{a}} u-\lambda u^{+}=g \quad \text { на } \Gamma .
$$

Здесь $u^{+}=\left.u\right|_{\Gamma}$ и $\partial_{\nu_{a}}-$ конормальная производная на $Г$. На гладких функциях

$$
\partial_{\nu_{a}} u(x)=\sum \nu_{j} a_{j k}(x) \partial_{k} u(x)
$$

где $\nu_{j}$ - координаты единичной внешней нормали к Г (существующей почти всюду на Г). В общем случае конормальная производная определяется формулой Грина

$$
(L u, v)_{\Omega}=\Phi(u, v)-\left(\partial_{\nu_{a}} u, v^{+}\right)_{\Gamma} .
$$

Подставив сюда $\partial_{\nu_{a}} u$ из (4.2.1), получим, поскольку $L u=0$,

$$
\Phi(u, v)-\lambda\left(u^{+}, v^{+}\right)_{\Gamma}=\left(g, v^{+}\right)_{\Gamma} .
$$

Пусть $u=v$ и $\mu=-\operatorname{Re} \lambda$. Тогда при $\mu \geqslant \mu_{0}$ с достаточно большим $\mu_{0}$

$$
\operatorname{Re} \Phi(u, u)+\mu\left(u^{+}, u^{+}\right)_{\Gamma}>0
$$

1) Полнота имеет место также в «промежуточных» пространствах, в частности в $L_{p}(\Omega)$. 
и это исходный пункт: наша задача - с дискретным спектром, лежащим в правой полуплоскости и, может быть, окрестности начала.

В центре квадрата $Q$ у нас $u, v \in H^{1}(\Omega)$, граничные значения $u^{+}, v^{+} \in$ $H^{1 / 2}(\Gamma)$ и $\partial_{\nu_{a}} u, g \in H^{-1 / 2}(\Gamma)$. В остальных точках прямоугольника $Q_{\delta}$

$$
\begin{gathered}
u \in H_{p}^{1 / 2+s+1 / p}(\Omega), \quad v \in H_{p^{\prime}}^{1 / 2-s+1 / p^{\prime}}(\Omega), u^{+} \in B_{p}^{1 / 2+s}(\Gamma), \quad v^{+} \in B_{p^{\prime}}^{1 / 2-s}(\Gamma), \\
w=\partial_{\nu_{a}} u, \quad g \in B_{p}^{-1 / 2+s}(\Gamma) .
\end{gathered}
$$

Операторы перехода от $u$ к $u^{+}$и от $v$ к $v^{+}$ограничены и имеют ограниченные правые обратные [22]. Функции $g$ и $v^{+}-$в сопряженных пространствах. В $\Omega$ можно пользоваться и пространствами Бесова вместо пространств потенциалов [22]. Функционал $\left(g, v^{+}\right)_{\Gamma}$ можно записать в виде $(h, v)_{\Omega}$ с некоторым $h \in \widetilde{H}_{p}^{-1 / 2+s-1 / p^{\prime}}(\Omega)$. При этом

$$
\left|\left(g, v^{+}\right)\right| \leqslant C_{1}\|g\|_{B_{p}^{-1 / 2+s}(\Gamma)}\left\|v^{+}\right\|_{B_{p^{\prime}}^{1 / 2-s}(\Gamma)} \quad \text { и } \quad\left\|v^{+}\right\|_{B_{p^{\prime}}^{1 / 2-s}(\Gamma)} \leqslant C_{2}\|v\|_{H_{p^{\prime}}^{1 / 2-s+1 / p^{\prime}}(\Omega)}
$$

для любой $v \in H_{p^{\prime}}^{1 / 2-s+1 / p^{\prime}}(\Omega)$, поэтому

$$
\|h\|_{\widetilde{H}_{p}^{-1 / 2+s-1 / p^{\prime}}(\Omega)} \leqslant C_{3}\|g\|_{B_{p}^{-1 / 2+s}(\Gamma)} .
$$

С учетом оценок в $\S 2$ мы получаем равномерные по $\lambda$ оценки для решения

$$
\|u\|_{B_{p}^{1 / 2+s}(\Gamma)} \leqslant C_{4}\|u\|_{H_{p}^{1+s+1 / p}(\Omega)} \leqslant C_{5}\|h\|_{\widetilde{H}_{p}^{-1 / 2+s-1 / p^{\prime}}(\Omega)} \leqslant C_{6}\|g\|_{B_{p}^{-1 / 2+s}(\Gamma)} .
$$

В силу (4.2.4)

$$
\mu \sup _{v^{+}} \frac{\left|\left(u^{+}, v^{+}\right)_{\Gamma}\right|}{\left\|v^{+}\right\|_{B_{p^{\prime}}^{1 / 2-s}(\Gamma)}} \leqslant \sup _{v^{+}} \frac{|\Phi(u, v)|}{\left\|v^{+}\right\|_{B_{p^{\prime}}^{1 / 2-s}(\Gamma)}}+\sup _{v^{+}} \frac{\left|\left(g, v^{+}\right)_{\Gamma}\right|}{\left\|v^{+}\right\|_{B_{p^{\prime}}^{1 / 2-s}(\Gamma)}}
$$

что в силу $(1.2 .4),(4.2 .9)$ и ограниченности оператора $v^{+} \mapsto v$ дает

$$
\mu\left\|u^{+}\right\|_{B_{p}^{-1 / 2+s}(\Gamma)} \leqslant C_{7}\|g\|_{B_{p}^{-1 / 2+s}(\Gamma)} \cdot
$$

В итоге видно, что мы имеем ограниченный оператор, переводящий данные Неймана $w=\partial_{\nu_{a}} u$ в данные Дирихле $u^{+}$,

$$
\mathscr{N}: B_{p}^{-1 / 2+s}(\Gamma) \rightarrow B_{p}^{1 / 2+s}(\Gamma),
$$

с оценкой $\|\mathscr{N} w\|_{B_{p}^{1 / 2+s}(\Gamma)}+\mu\|\mathscr{N} w\|_{B_{p}^{-1 / 2+s}(\Gamma)} \leqslant C_{8}\|w\|_{B_{p}^{-1 / 2+s}(\Gamma)}$. Обратный оператор, обозначим его $\mathscr{D}$, действует из $B_{p}^{1 / 2+s}(\Gamma)$ в $B_{p}^{-1 / 2+s}(\Gamma)$, он имеет компактную в $B_{p}^{-1 / 2+s}(\Gamma)$ резольвенту с оптимальной оценкой, которую мы перепишем в $\Theta(r, \varphi)$ в виде

$$
\left\|R_{\mathscr{D}}(\lambda)\right\| \leqslant C_{9}(1+|\lambda|)^{-1}
$$

Здесь возникает вторая конкретная реализация для абстрактной ситуации, рассмотренной в $\S 3$, с $X=B_{p}^{-1 / 2+s}(\Gamma), Y=B_{p}^{1 / 2+s}(\Gamma)$. Асимптотика собственных чисел самосопряженных задач (в смысле $(\mathscr{D} \varphi, \psi)_{\Gamma}=(\varphi, \mathscr{D} \psi)_{\Gamma}$ на $\left.H^{1 / 2}(\Gamma)\right)$ в общем случае не получена, известны только частичные результаты [40], [4]. Но для оператора $J$ вложения $Y$ в $X$ есть оценка $a$-чисел: $a_{k}(J)=O\left(k^{-1 /(n-1)}\right)$. 
Она выводится из предложения 3.1.1 и из свойства $2^{\circ}$-ч-чисел при помощи разбиения единицы на $Г$. Для оператора $N=J \mathscr{N}$, используя свойство $9^{\circ}$ в центре квадрата, получаем оценку $\lambda_{k}(N)=O\left(k^{-1 /(n-1)}\right)$. Для оператора $(1-\lambda N)^{-1}$ получаем представление в виде отношения целых функций, операторнозначной и числовой, роста не выше чем порядка $n-1$ и нормального типа. Условие на угол в (1.1.13):

$$
2(n-1) \varphi<\pi .
$$

Снова можно проверить независимость собственных значений и корневых функций от $(s, t) \in Q_{\delta}$ и вывести полноту из известных результатов для $p=2$, а также получить теорему о суммируемости для $p=2$. См. [5]. Но окончательная теорема получается с использованием результатов $\S 3$.

Теорема 4.2.1. Собственные значения $\lambda_{k}(N)$ оператора $N$ не зависят от $(s, t) \in Q_{\delta}$ и имеют оценку $O\left(k^{-1 /(n-1)}\right)$. Корневые функиии также не зависят om $(s, t) \in Q_{\delta}$ и принадлежсат пересечению пространств Бесова $B_{p}^{1 / 2+s}(\Gamma)$. Oператор $(I-\lambda N)^{-1}$ представим в виде отношения целых аналитических функиий, операторнозначной и числовой; функиия в знаменателе - роста не выше чем порядка $n-1$ и нормального типа; норма числителя имеет аналогичную оценку. Если выполнено условие (1.1.4), обеспечивающее оптимальную оценку резольвенты в $\Theta(r, \varphi)$, где ч подчинено условию (4.2.13), то имеет место полнота корневых функиий в пространствах $B_{p}^{1 / 2+s}(\Gamma)$ u $B_{p}^{-1 / 2+s}(\Gamma)$, а при $n-1<q<\pi /(2 \varphi)$ - суммируемость рядов Фуръе по ним методом Абеля-Лидского порядка q в этих пространствах.

Аналоги результатов этого параграфа справедливы для «гладких» эллиптических с параметром задач в банаховых пространствах потенциалов и Бесова; к сожалению, здесь на этом не удастся остановиться.

\section{§5. Смешанные задачи для параболических уравнений}

В этом параграфе $t$ - время, и обозначением $t=1 / p$ мы здесь не будем пользоваться. Буквы $g$ и $v$ также имеют новый смысл. Рассмотрим уравнение

$$
\partial_{t} v(t, x)=A v(t, x)+g(t, x)
$$

в липшицевом цилиндре $[0, \infty) \times \Omega$ с начальным условием

$$
v(0, x)=v_{0}(x)
$$

и однородными условиями Дирихле или Неймана на боковой поверхности $[0, \infty)$ $\times \Gamma$. Здесь мы полагаем $A=-L$, чтобы приблизиться к обозначениям в [31]. Пример: $\partial_{t} v=\Delta v+g$.

Мы предположим, что для оператора $L$ выполнено условие (1.1.3) сильной эллиптичности. Тогда его можно усилить до (1.1.4), поэтому есть параболическая (или аналитическая) полугруппа, решающая задачу, и эта задача однозначно разрешима в указанном ниже смысле.

Точный результат для абстрактных операторов состоит в следующем ([31], гл. 5, §4). Пусть $X$ - банахово пространство с нормой $\|x\|=\|x\|_{X}$ и $A-$ замкнутый оператор в $X$ с плотной областью определения $Y=\mathscr{D}(A)$. (B $Y$ можно ввести норму графика $\left.\|x\|_{Y}=\|x\|+\|A x\|.\right)$ Введем множество

$$
\Sigma(\theta, r)=\{\lambda:|\arg \lambda| \leqslant \varphi,|\lambda| \geqslant r\}
$$


с $\varphi>\pi / 2$. Предположим, что оно лежит в резольвентном множестве оператора $A$ и там выполнена оценка

$$
\left\|(A-\lambda I)^{-1}\right\| \leqslant C_{1}(1+|\lambda|)^{-1}
$$

с не зависящей от $\lambda$ постоянной $C_{1}$. Введем операторы

$$
S_{t} v=\frac{1}{2 \pi i} \int_{\Gamma_{\Sigma}} e^{\lambda t}(\lambda I-A)^{-1} v d \lambda \quad(t>0, v \in X),
$$

где $\Gamma_{\Sigma}$ - контур множества (5.1.3) с направлением обхода снизу вверх, как в п. 3.5. Интеграл (5.1.5) похож на интеграл (3.5.3) с $q=1$.

Это по сути обратное преобразование Лапласа от $(\lambda I-A)^{-1}$ (или экспонента $\exp (t A))$. В [31] доказывается, что эти операторы обладают следующими свойствами. (Свойствами 1 и 3 обладает и интеграл (3.5.3).)

1. Они действуют из $X$ в $Y$ при $t>0$ и удовлетворяют в $X$ оценке $\left\|S_{t}\right\| \leqslant C$ с некоторой постоянной $C$.

2. Имеет место полугрупповое свойство $S_{t+\tau}=S_{t} S_{\tau}(t, \tau>0)$.

3. Имеет место сильная сходимость $\lim _{t \rightarrow+0} S_{t} v=v(v \in X)$.

4. $\lim _{t \rightarrow+0}\left(S_{t} v-v\right) / t=A v(v \in Y)$, так что $A-$ инфинитезимальный производящий оператор для этой полугруппы.

Если $v_{0} \in Y$ и $g(t)$ - функция на $\mathbb{R}_{+}$со значениями в $X$, удовлетворяющая условию Гёльдера $\left\|g(t)-g\left(t^{\prime}\right)\right\| \leqslant C_{2}\left|t-t^{\prime}\right|^{\eta}, \eta \in(0,1]$, то задача Коши

$$
\partial_{t} v(t)=A v(t)+g(t) \quad(t>0), \quad v(0)=v_{0}
$$

имеет решение $v(t)$. Это функция, определяемая формулой

$$
v(t)=S_{t} v_{0}+\int_{0}^{t} S_{t-s} g(s) d s,
$$

и это решение единственно. Оно голоморфно по $t$ в малом угле на комплексной плоскости с биссектрисой $\mathbb{R}_{+}$.

У нас в случае граничных условий Неймана $X=\widetilde{H}_{p}^{-1 / 2+s-1 / p^{\prime}}(\Omega), Y=$ $H_{p}^{1 / 2+s+1 / p}(\Omega)$, где $|s|<1 / 2,|t-1 / 2|<\delta$, и эти пространства можно заменить соответствующими пространствами Бесова. В случае задачи Дирихле тильда переходит с первого на второе пространство.

Мы не будем вдаваться здесь в дальнейшее исследование решений этой и других нестационарных задач.

\section{§6. Обобщения на системы}

Все сказанное выше, кроме п. 4.2, обобщается на рассмотренные в [6] системы с параметром, в которых все числа $m_{j}$ одинаковы. (К более общему случаю мы предполагаем вернуться в другом месте.) На систему накладывается дополнительное условие неотрицательности (или выпуклости) алгебраической формы, связанной с главным символом, а в случае задачи Неймана - и соответствующее условие коэрцитивности (см. п. 2.1 в [6]). Число $\gamma$ равно $2 m / n$, где $2 m-$ порядок системы. При рассмотрениях из п. 4.2 и 55 для простоты дополнительно предполагаем, что система - второго порядка $(m=1)$. 
Отметим, что в ряде работ параболические уравнения и системы в липшицевых цилиндрах исследовались при помощи потенциалов, см., в частности, [13], [32] и приведенные там ссылки.

\section{ЛитерАТУРА}

[1] Sh. Agmon, On the eigenfunctions and on the eigenvalues of general elliptic boundary value problems, Comm. Pure Appl. Math., 15:2 (1962), 119-147.

[2] М. С. Агранович, Спектральные свойства задач дифракиии, в кн.: Н. Н. Войтович, Б. З. Каценеленбаум, А. Н. Сивов, Обобщенный метод собственных колебаний в теории дифракции, Наука, М., 1977, 289-416; Английское переработанное издание: M. S. Agranovich, B. Z. Katsenelenbaum, A. N. Sivov, N. N. Voitovich, Generalized Method of Eigenoscillations in Diffraction Theory, Wiley-VCH, Berlin etc., 1999, Chapter V.

[3] М. С. Агранович, Спектральные задачи для сильно эллиптических систем второго порядка в областях с гладкой и негладкой гранищей, УМН, 57:5 (2002), 3-78.

[4] M. S. Agranovich, On a mixed Poincaré-Steklov type spectral problem in a Lipschitz domain, Russ. J. Math. Phys., 13:3 (2006), 239-244.

[5] М. С. Агранович, Регулярность вариащионных решений линейных граничных задач в липшицевых областях, Функц. анализ и его прил., 40:4 (2006), 83-103.

[6] М. С. Агранович, $K$ теории задач Дирихле и Неймана для линейных сильно эллиптических систем в липшицевых областях, Функц. анализ и его прил., 41:4 (2007), 1-21.

[7] M. S. Agranovich, Remarks on potential spaces and Besov spaces in a Lipschitz domain and on Whitney arrays on its boundary, Russ. J. Math. Phys., 15:2 (2008), 146-155.

[8] М. С. Агранович, М. И. Вишик, Эллиптические задачи с параметром и параболические задачи общего вида, УМН, 19:3 (1964), 53-161.

[9] Й. Берг, Й. Лёфстрём, Интерполяционные пространства, Мир, М., 1980.

[10] М. С. Бирман, М. З. Соломяк, Кусочно-полиномиалъные приближения функиий классов $W_{p}^{\alpha}$, Матем. сб., 73:3 (1967), 331-355.

[11] М. С. Бирман, М. З. Соломяк, Спектральная асимптотика негладких эллиптических операторов, I, II, Труды ММО, 27 (1972), 3-52; 28 (1973), 3-34.

[12] М. С. Бирман, М. З. Соломяк, Количественный анализ в теоремах вложения Соболева и приложения $\kappa$ спектралъной теории, в кн.: Десятая матем. школа, Ин-т математики АН УССР, Киев, 1974, 5-189.

[13] R. M. Brown, Z. Shen, A note on boundary value problems for the heat equation in Lipschitz cylinders, Proc. Amer. Math. Soc., 119:2 (1993), 585-594.

[14] J. Burgoyne, Denseness of the generalized eigenvectors of a discrete operator in a Banach space, J. Operator Theory, 33 (1995), 279-297.

[15] Н. Данфорд, Дж. Т. Шварц, Линейные операторы, т. II, Мир, М., 1966.

[16] D. E. Edmunds, W. D. Evans, Spectral Theory and Differential Operators, Clarendon Press, Oxford Univ. Press, Oxford, 1987.

[17] D. E. Edmunds, H. Triebel, Function Spaces, Entropy Numbers, and Differential Operators, Cambridge Univ. Press, Cambridge, 1996.

[18] И. Ц. Гохберг, М. Г. Крейн, Введение в теорию несамосопряженных операторов в гильбертовом пространстве, Наука, М., 1965.

[19] A. Grothendieck, Produits tenzoriels topologiques et espaces nucléares, Mem. Amer. Math. Soc., 16 (1955).

[20] А. Гротендик, Теория Фредгольма, Сб. переводов Математика, 2:5 (1958), 51-103.

[21] S. Janson, P. Nilsson, J. Peetre, Notes on Wolff's note on interpolation spaces, Proc. London Math. Soc., 48:2 (1984), 283-299. 
[22] A. Jonsson, H. Wallin, Function Spaces on Subsets of $\mathbb{R}^{n}$, Math. Rep., Ser. 2, no. 1, Academic Publ., Harwood, 1984.

[23] H. König, Eigenvalue Distribution of Compact Operators, Operator Theory: Advances and Applications, vol. 16, Birkhäuser, Basel etc., 1986.

[24] V. A. Kozlov, V. G. Maz'ya, J. Rossmann, Elliptic Boundary Value Problems in Domain with Point Singularities, Amer. Math. Soc., Providence, RI, 1997.

[25] P. D. Lax, A. N. Milgram, Parabolic equations, in: Contributions to the Theory of Partial Differential Equations, Ann. of Math. Studies, vol. 33, Princeton Univ. Press, Princeton, NJ, 1954, 167-190.

[26] Б. Я. Левин, Распределение корней цельх функиий, Гостехиздат, М., 1956.

[27] В. Б. Лидский, О суммируемости рядов по главным векторам несамосопряженных операторов, Труды ММО, 11 (1962), 3-35.

[28] А. С. Маркус, Некоторые признаки полноты системы корневых векторов линейного оператора в банаховом пространстве, Матем. сб., 70 (112):4 (1966), 526-561.

[29] А. С. Маркус, В. И. Мацаев, Аналоги неравенств Вейля и теоремы о следе в банаховом пространстве, Матем. сб., 86:2 (1971), 299-313.

[30] В. И. Мацаев, Об одном методе оценки резолъвент несамосопряженных операторов, Докл. АН СССР, 154:5 (1964), 1034-1037.

[31] С. Мизохата, Теория уравнений с частными производными, Мир, М., 1977.

[32] M. Mitrea, The initial Dirichlet boundary value problem for general second order parabolic systems in nonsmooth manifolds, Comm. Partial Differential Equations, 26:11-12 (2001), 1975-2036.

[33] M. Mitrea, M. Taylor, Potential theory on Lipschitz domains in Riemannian manifolds: Sobolev-Besov space results and the Poisson problem, J. Funct. Anal., 176:1 (2000), 1-79.

[34] L. Nirenberg, Remarks on strongly elliptic partial differential equations, Comm. Pure Appl. Math., 8 (1955), 649-675.

[35] A. Pietsch, Eigenvalues and s-Numbers, Acad. Verl., Leipzig, 1987; Cambridge Studies in Adv. Math., vol. 13, Cambridge University Press, Cambridge, 1987.

[36] V. S. Rychkov, On restrictions and extensions of the Besov and Triebel-Lizorkin spaces with respect to Lipschitz domains, J. London Math. Soc. (2), 60:1 (1999), 237-257.

[37] J. Savaré, Regularity results for elliptic equations in Lipschitz domains, J. Funct. Anal., 152:1 (1998), 176-201.

[38] Z. Shen, Resolvent estimates in $L^{p}$ for elliptic systems in Lipschitz domains, J. Funct. Anal., 133:1 (1995), 224-251.

[39] И. Я. Шнейберг, Спектральные свойства линейных операторов в интерполяиионных семействах банаховых пространств, Матем. исслед., 9:2 (1974), 214-227.

[40] T. A. Suslina, Spectral asymptotics of variational problems with elliptic constraints in domains with piecewise smooth boundary, Russ. J. Math. Phys., 6:2 (1999), 214-234.

[41] Х. Трибель, Теория интерполяции, функииональные пространства, дифберенциальные операторы, Мир, М., 1980.

[42] H. Triebel, Function spaces in Lipschitz domains and on Lipschitz manifolds. Characteristic functions as pointwise multipliers, Rev. Mat. Comput., 15:2 (2002), 475-524.

[43] М. И. Вишик, О сильно эллиптических системах дифференциальных уравнений, Матем. сб., 29 (71):3 (1951), 615-676.

Московский институт электроники и математики

Поступило в редакцию e-mail: magran@orc.ru 28 мая 2008 г. 